\title{
On the QSO Content of the First Byurakan Survey and the Completeness of the Palomar Green Survey
}

\author{
P. Véron ${ }^{1}$, A. M. Mickaelian ${ }^{2}$, A. C. Gonçalves ${ }^{1}$ and M.-P. Véron-Cetty ${ }^{1}$ \\ ${ }^{1}$ Centre National de la Recherche Scientifique, Observatoire de Haute \\ Provence, 04870 St. Michel l'Observatoire, France \\ ${ }^{2}$ Byurakan Astrophysical Observatory, Byurakan 378433, Republic of \\ Armenia
}

\begin{abstract}
The second part of the First Byurakan Survey is aimed at detecting all bright $(B<16.5)$ UV-excess starlike objects in a large area of the sky. By comparison with major X-ray and radio surveys we tentatively identified as QSOs 11 FBS objects. We made spectroscopic observations of nine of them. We found six new QSOs bringing the total number of known QSOs in this survey to 42 .

By comparison with the Palomar Green (PG) QSO survey, we found that the completeness of this last survey is of the order of $70 \%$ rather than $30-50 \%$ as suggested by several authors.
\end{abstract}

\section{Introduction}

The surface density of bright QSOs $(B<17.0)$ is still very poorly known. The PG survey (Schmidt \& Green 1983) covering an area of $10714 \mathrm{deg}^{2}$ led to the discovery of 69 QSOs brighter than $M_{B}=-24\left(H_{\mathrm{o}}=50 \mathrm{~km} \mathrm{~s}^{-1} \mathrm{Mpc}^{-1}\right)$ and $B=$ 16.16 , corresponding to $0.0064 \mathrm{deg}^{-2}$. However, several authors have concluded that it could be incomplete by a factor 2 to 3 .

The First Byurakan Survey (FBS), also known as the Markarian survey, was carried out in 1965-80 by Markarian et al. (1989). It covers $17000 \mathrm{deg}^{2}$ and is complete to about $B=16.5^{\mathrm{m}}$. It has been used by Markarian and his collaborators to search for UV excess galaxies; it can also be used for finding UV-excess starlike objects. Such a program - the second part of the FBS has been undertaken in 1987 (Abrahamian et al. 1990; Mickaelian 1994). Its main purpose is to take advantage of the large sky area covered to get a reliable estimate of the surface density of bright QSOs.

At the present time, $4109 \mathrm{deg}^{2}$ have been searched and a catalogue of 1103 blue stellar objects $\left(715\right.$ at $|b|>30^{\circ}$ ) has been built. 434 spectroscopic identifications (398 stars and 36 QSOs) are already known. Photoelectric UBV photometry for $113 \mathrm{FBS}$ objects has been published. For all except one, $U-B$ $<-0.50$. 


\section{Comparison with $\mathrm{X}$-ray and radio surveys}

\subsection{The ROSAT All-sky Survey Bright Source Catalogue (RASS- BSC)}

We have cross-correlated the list of 1103 UV-excess objects with the RASSBSC (Voges et al. 1996). We have found seven coincidences with FBS objects of unknown nature.

\subsection{The WGA ROSAT catalogue of point sources (WGACAT)}

The WGACAT has been generated using the ROSAT PSPC pointed data publicly available as of September 1994. It contains more than 45600 individual sources (White et al. 1994). We have found two coincidences with FBS objects of unknown nature.

\subsection{The NRAO VLA sky survey (NVSS)}

The NVSS covers the sky north $\delta=-40^{\circ}$ at $1.4 \mathrm{GHz}$ (Condon et al. 1998). It contains almost $2 \times 10^{6}$ discrete sources stronger than $\mathrm{S}=2.5 \mathrm{mJy}$. We have found two coincidences with FBS objects of unknown nature.

\section{Observations}

Spectroscopic observations of nine of the eleven objects coincident either with a X-ray or a radio source were carried out with the Observatoire de Haute Provence $1.93 \mathrm{~m}$ telescope. Six (listed in Table 1; the spectra are shown in Fig. 1) turned out to be QSOs, while three are stars.

\section{Discussion}

\subsection{Completeness of the FBS}

The Catalogue of mean UBV data on stars (Mermilliod \& Mermilliod 1994) contains 102 stars in the FBS area, with $11.0<V<16.5$ (bright stars are saturated on the FBS plates and are therefore missed) and $U-B<-0.50 ; 53$ are included in the FBS catalogue suggesting that, in this magnitude interval, the completeness of the FBS is $52 \%(53 / 102)$. The completeness of the survey increases from $\sim 20 \%$ at $U-B \sim-0.6$ to $\sim 80 \%$ for $U-B<-1.0$. The QSO $U-B$ colour changes with $z$; but most of the changes, at least for $z<2.2$, are due to the presence of an emission line in one of the two filters. The $U-B$ colour of the continuum is in the range $-0.9<U-B<-0.7$. Slitless spectroscopic surveys are sensitive to the colour of the continuum, unaffected by the emission lines. There are 58 known stars with $14.0<V<16.5$ and $U-B<-0.70$ in the FBS area; 39 (67\%) have been found by the FBS; we shall adopt this value for the completeness of the FBS.

The PG and FBS samples have about $2250 \mathrm{deg}^{2}$ in common. Out of the 1103 FBS objects, 618 are within the PG fields, 276 being in the PG sample. Thirty-six PG objects have not been found in the FBS. So $88 \%(276 / 312)$ of the PG objects have been discovered. The 36 undiscovered objects have been 

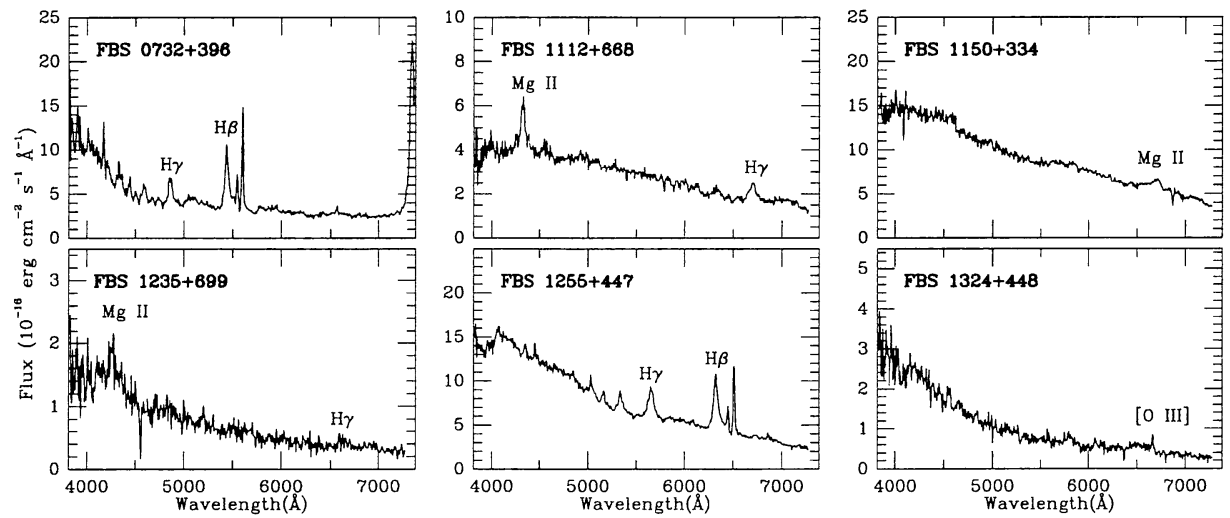

Figure 1. Spectra of the six newly discovered QSOs.

examined on the FBS plates; 24 have a weak UV excess (the PG survey finds a significant fraction of stars with $U-B \sim-0.4$, while the FBS is relatively insensitive for $U-B>-0.7)$. From this, we conclude that the FBS is $\sim 95 \%$ complete for $U-B<-0.5$. This is significantly larger than the $67 \%$ success rate obtained from the UBV stars; it is probably due to the fact that, in principle, the PG survey contains only objects brighter than $B=16.2$. There are $25 \mathrm{PG}$ QSOs in the FBS area, 23 of them have been found, confirming that the FBS is very efficient in discovering bright QSOs.

Table 1. Newly discovered QSOs

\begin{tabular}{lcccccccl}
\hline FBS name & Mag. & $O$ & & $(1)$ & $(2)$ & & $b$ & \multicolumn{1}{c}{$z$} \\
\hline $0732+396$ & 16.0 & 14.70 & $\mathrm{X}$ & 10 & 20 & $\mathrm{~N}$ & 25.1 & 0.118 \\
$1112+668$ & 17.0 & 16.53 & $\mathrm{X}$ & 10 & 4 & $\mathrm{Y}$ & 47.9 & 0.544 \\
$1150+334$ & 16.2 & 16.30 & $\mathrm{R}$ & 0.8 & 1.2 & $\mathrm{Y}$ & 76.0 & 1.40 \\
$1235+699$ & 17.9 & 17.96 & $\mathrm{X}$ & 5 & 4 & $\mathrm{Y}$ & 47.4 & $0.522 ?$ \\
$1255+447$ & 16.5 & 16.48 & $\mathrm{X}$ & 10 & 13 & $\mathrm{Y}$ & 72.6 & 0.300 \\
$1324+448$ & 17.0 & 18.09 & $\mathrm{X}$ & 8 & 9 & $\mathrm{Y}$ & 71.1 & $0.331 ?$ \\
\hline \hline
\end{tabular}

$\mathrm{X}$ : in the RASS-BSC; $\mathrm{x}$ : in the WGACAT; R: in the NVSS; (1): error of the ROSAT or VLA position (in arcsec); (2): distance between the FBS and ROSAT or VLA positions (in arcsec); $Y$ : in the $P G$ area; $N$ : not in the PG area; $O$ : APS $O$ magnitudes.

\subsection{The AGN content of the FBS}

Thirty-four FBS objects are listed as QSOs in the Véron-Cetty \& Véron (1998) catalogue. Two more have been shown to be QSOs by Hagen et al. (1998) and six have been identified in the present paper. There are therefore 42 known QSOs in the FBS, 41 being at high galactic latitude $\left(|b|>30^{\circ}\right)$. At high galactic latitude, all FBS objects associated with a RASS-BSC source have been identified. Among them, there are 25 QSOs. As about $60 \%$ of all PG QSOs are RASS sources, and assuming that this is true for the FBS QSOs, we should have a total of about 43 QSOs in the FBS catalogue. This suggests that the number of QSOs still to be found in the FBS catalogue is very small as 42 are already known. 
All 114 AGNs from the PG survey have been observed at $5 \mathrm{GHz}$ with the VLA (Kellerman et al. 1989); $35(30 \%)$ have been detected with a flux density larger than $3 \mathrm{mJy}$. The same fraction $(12 / 40)$ of the known FBS QSOs have been detected in the NVSS survey, confirming that the number of QSOs in the as yet spectroscopically unobserved FBS objects is small and probably cannot exceed 10, as the fraction of radio-detected QSOs would then drop below $25 \%$ and be significantly lower than the corresponding fraction for the PG survey.

\subsection{Completeness of the PG survey}

In principle the PG survey selected all objects with $U-B<-0.46$ and brighter than $B \sim 16.2$; however, the $U-B$ colour was measured with a relatively large error $\left(0.24^{\mathrm{m}} \mathrm{rms}\right)$ which induced an incompleteness estimated at around $12 \%$. Moreover, in the interval $0.6<z<0.8$, the strong $\mathrm{Mg}$ II $\lambda 2800$ emission line is in the $B$ filter which gives a much redder $U-B$ colour than for neighbouring redshifts, resulting in an incompleteness of $28 \%$ in this range (Schmidt \& Green 1983).

The catalogue of mean UBV data on stars (Mermilliod \& Mermilliod 1994) contains 283 stars in the magnitude range $12.0<B<16.5$ and with $U-B<$ -0.40 in the full $10714 \mathrm{deg}^{2}$ area of the PG survey; 190 are included in the PG catalogue. Twenty-four stars photoelectrically observed because they were in the PG catalogue have been ignored; the overall completeness of the PG survey is therefore $64 \%(166 / 259) .67 \%(162 / 241)$ of the stars brighter than $B=16.2$ were found in the PG survey, while only $22 \%(4 / 18)$ of the weaker stars were. The completeness of the PG survey rises from about $55 \%$ at $U-B=-0.60$ to $80 \%$ at $U-B<-1.0$. For PG QSOs, the completeness should not be less than $\sim 70 \%$.

An independent estimate of the completeness of the PG survey comes from the comparison with the RASS-BSC. Of the 25 PG QSOs in the FBS area, 13 are brighter than $B=16.16$ and have been detected by ROSAT. Twelve of them have been found in the FBS. All FBS objects associated with a ROSAT source, located in the PG area, have been spectroscopically identified; among them, there are eleven non-PG QSOs. No more than two are suspected to have been possibly bright enough to have been detected by the PG survey. This suggests a completeness of about $87 \%(13 / 15)$ with however a large uncertainty due to the smallness of the sample.

Goldschmidt et al. (1992), Köhler et al.(1997) and La Franca \& Cristiani (1997) have claimed that the PG survey is incomplete by a factor of 2 to 3 , but their samples are quite small (5, 8 and 7 objects, respectively); in addition, we do not know if there is an offset between their magnitude scales and that of the PG survey (the mean differences between the PG and photoelectric $B$ magnitudes for 190 stars is equal to $-0.02^{\mathrm{m}}$ ). Therefore, their results should be considered as tentative.

\section{Building a "complete" QSO survey based on APS O magnitudes}

Because of their variability, it is an impossible task to compare directly two QSO surveys of the same region of the sky made at different epochs. However we now have, for a large fraction of the sky, the possibility to extract from the 
APS database, for any object, the $O$ magnitude as measured on the Palomar Sky Survey plates with an accuracy of about $0.2^{\mathrm{m}}$ (Pennington et al. 1993). By doing this for all known QSOs found in the course of a number of different surveys of the same area of the sky, we may hope to reach as near as possible from an ideal survey complete to a well defined limiting magnitude.

We have extracted the APS $O$ magnitudes, when available, for all objects in the QSO catalogue (Véron-Cetty \& Véron 1998) brighter than $B=17$, with $M_{B}<-24.0$ and $z<2.15$, located in the $2400 \mathrm{deg}^{2}$ of the FBS at $|b|>30^{\circ}$. Whenever this $O$ magnitude exists, we give it the preference. There are 18 such QSOs with $O<16.0$ (from a sample of 105 UV-excess stars, we have found that $\langle\mathrm{O}-\mathrm{B}\rangle=-0.17$ with a rms error of $0.25^{\mathrm{m}}$ ), and seven with $B<16.2$. Thus our "complete" sample contains between 18 and 25 QSOs brighter than $B=$ 16.2 , or 0.0075 to $0.010 \mathrm{deg}^{-2}$, i.e. 1.2 to 1.6 times larger than the PG surface density. It should be possible, when the APS database will be completed, to get the $O$ magnitudes of the seven objects for which they are not yet available.

Acknowledgments. A. M. Mickaelian is grateful to the CNRS for making possible his visit to OHP. A.C. Gonçalves acknowledges the Fundação para $a$ Ciência e a Tecnologia, Portugal (PRAXIS XXI/BD/5117/95 PhD. grant).

\section{References}

Abrahamian, H. V., Lipovetsky, V.A. \& Stepanian, J. A. 1990, Astrophysics, 32, 14

Condon, J.J., Cotton, W.D., Greisen, E.W. et al. 1998, AJ, 115, 1693

Goldschmidt, P., Miller, L., La Franca, F. \& Cristiani, S. 1992, MNRAS, 256, $65 \mathrm{P}$

Hagen, H.-J., Engels, D. \& Reimers, D. 1998, A\&AS, (in press)

Kellerman, K. I., Sramek, R., Schmidt, M., Shaffer, D. B. \& Green, R. 1989, AJ, 98,1195

Köhler, T., Groote, D., Reimers, D. \& Wisotzki, L. 1997, A\&A, 325, 502

La Franca, F. \& Cristiani, S. 1997, AJ, 113, 1517

Markarian, B. E., Lipovetsky, V.A., Stepanian, J.A., Erastova, L. K. \& Shapovalova A. I. 1989, Commun. Special Astrophys. Obs., 62, 5

Mermilliod, J.-C. \& Mermilliod, M. 1994, Catalogue of mean UBV data on stars, Springer-Verlag

Mickaelian, A. M. 1994, Discovery and Investigation of Blue Stellar Objects of the First Byurakan Survey, Ph.D. thesis, Byurakan

Pennington, R. L., Humphreys, R. M., Odewahn, S. C., Zumach, W. \& Thurmes, P. M. 1993, PASP, 105, 521

Schmidt, M. \& Green, R.F. 1983, ApJ, 269, 352

Véron-Cetty, M.-P. \& Véron, P. 1998, ESO Scientific Report Nº18

Voges, W., Aschenbach, B., Boller, T. et al. 1996, IAU circ. 6420

White, N.E., Giommi, P. \& Angelini, L. 1994, IAU Circ. 6100 